\title{
CLOSED SUBGROUPS OF LATTICE-ORDERED PERMUTATION GROUPS
}

\author{
BY
}

\author{
STEPHEN H. MCCLEARY
}

\begin{abstract}
Let $G$ be an $l$-subgroup of the lattice-ordered group $A(\Omega)$ of order-preserving permutations of a chain $\Omega$; and in this abstract, assume for convenience that $G$ is transitive. Let $\bar{\Omega}$ denote the completion by Dedekind cuts of $\Omega$. The stabilizer subgroups $G_{\bar{\omega}}=\{g \in G \mid \bar{\omega} g=\bar{\omega}\}, \bar{\omega} \in \bar{\Omega}$, will be used to characterize certain subgroups of $G$ which are closed (under arbitrary suprema which exist in $G$ ). If $\Delta$ is an $o \cdot b l o c k$ of $G$ (a nonempty convex subset such that for any $g \in G$, either $\Delta g=\Delta$ or $\Delta g \cap \Delta$ is empty), and if $\bar{\omega}=$ sup $\Delta, G_{\Delta}$ will denote $\{g \in G \mid \Delta g=\Delta\}=G_{\bar{\omega}}$; and the o-block system $\tilde{\Delta}$ consisting of the translates $\Delta g$ of $\Delta$ will be called closed if $G_{\Delta}$ is closed. When the collection of $o$-block systems is totally ordered (by inclusion, view. ing the systems as congruences), there is a smallest closed system $\mathcal{C}$, and all systems above $\mathcal{C}$ are closed. $\mathcal{C}$ is the trivial system (of singletons) iff $G$ is complete (in $A(\Omega)$ ). $G_{\bar{\omega}}$ is closed iff $\bar{\omega}$ is a cut in $\mathcal{C}$, i.e., $\bar{\omega}$ is not in the interior of any $\Delta \in \mathcal{C}$. Every closed convex $l$-subgroup of $G$ is an intersection of stabilizers of cuts in $\mathcal{C}$. Every closed prime subgroup $\neq G$ is either a stabilizer of a cut in $\mathcal{C}$, or else is minimal and is the intersection of a tower of such stabilizers. $L(\mathcal{C})=\bigcap\left\{G_{\Delta} \mid \Delta \in \mathcal{C}\right\}$ is the distributive radical of $G$, so that $G$ acts faithfully (and completely) on $\mathcal{C}$ iff $G$ is completely distributive. Every closed $l$-ideal of $G$ is $L(D)$ for some system $D$. A group $G$ in which every nontrivial $o$-block supports some $1 \neq g \in G$ (e.g., a generalized ordered wreath product) fails to be complete iff $G$ has a smallest nontrivial system $\tilde{\Delta}$ and the restriction $G_{\Delta} \mid \Delta$ is $o-2-t r a n s i t i v e$ and lacks elements $\neq 1$ of bounded support.

These results about permutation groups are used to show that if $H$ is an abstract $l$-group having a representing subgroup, its closed $l$-ideals form a tower under inclusion; and that if $\left\{K_{\lambda}\right\}$ is a Holland kernel of a completely distributive abstract $l-$ group $H$, then so is the set of closures $\left\{K_{\lambda}^{*}\right\}$, so that if $H$ has a transitive representation as a permutation group, it has a complete transitive representation.
\end{abstract}

1. Introduction. A permutation $f$ of a chain $\Omega$ is said to preserve order if $\beta \leq \gamma$ implies $\beta f \leq \gamma f$ for all $\beta, \gamma \in \Omega$. The group $A(\Omega)$ of all order-preserving permutations becomes a lattice-ordered group (l-group) when ordered pointwise. We shall treat losubgroups $G$ of $A(\Omega)$, i.e., subgroups which are also sublattices; these will be known as l-permutation groups. $G$ will be assumed transitive only

Presented to the Society, January 19, 1972; received by the editors November 19, 1971. AMS (MOS) subject classifications (1970). Primary 06A05, 06A55; Secondary 20F25.

Key words and phrases. Latticeordered permutation group, totally ordered set, complete subgroup, prime subgroup, closed subgroup, stabilizer subgroup, complete distributivity, wreath product. 
in $\$ \$ 4$ and 5. A convex losubgroup $P$ of an logroup $G$ is prime if $g_{1} \wedge g_{2} \in P$ implies $g_{1} \in P$ or $g_{2} \in P$. If $(G, \Omega)$ is an l-permutation group, each $g \in G$ can be uniquely extended to an order-perserving permutation of $\bar{\Omega}$ which we shall identify with $g$. For the extended group we shall write $(G, \bar{\Omega})$. Stabilizer subgroups $G_{\bar{\omega}}, \bar{\omega} \in \bar{\Omega}$, are prime.

Lloyd [7, Theorem 2] showed that in a rather restricted class of (possibly intransitive) l-permutation groups, the stabilizers $G_{\alpha}, \alpha \in \Omega$, are closed. In [9], the present author showed that for groups in that class, the stabilizers $G_{\bar{\omega}}, \bar{\omega} \epsilon$ $\bar{\Omega}$, constitute all closed primes $\neq G$. In preparation for the description in Theorem 9 of various closed subgroups of $G$, the present paper extends the information in [9], and the discussion in [8] of the conditions (1) All sups in $G$ are point wise, (2) $G$ is complete, (3) Stabilizers $G_{a}$ are closed, and (4) $G$ is completely distributive. However, dependence on these papers is kept to a minimum.

Several examples are given at the end of the paper, including one of a transo itive group (necessarily with an infinite number of o-block systems) with a closed prime which is not a stabilizer.

2. Prime subgroups of an logroup. If $P$ is a prime subgroup of an logroup $H$, then $\{Q \mid P \subseteq Q, Q$ prime in $H\}$ is totally ordered by inclusion [3, Lemma 3], and this tower contains all convex subgroups of $H$ which contain $P$.

Lemma 1 (Byrd and Lloyd [2, Lemma 3.3]). If $P$ and $Q$ are prime subgroups of an l.group $H$, with $P$ closed and $P \subseteq Q$, then $Q$ is also closed.

Another hypothesis that guarantees closure of a prime subgroup is

Proposition 2. Let $P$ be a prime subgroup of an l-group $H$ and let $Q$ be a convex l-subgroup of $H, Q \notin \mathbb{P}$. Then if $P \cap Q$ is closed in $H$, so is $P$.

Proof. Suppose $s=\sup \left\{s_{i} \mid i \in I\right\}$, with $1 \leq s_{i} \in P$ for all $i$. Pick $1<g \epsilon$ $Q \backslash P$. Then $q \wedge s=\sup \left\{q \wedge s_{i}\right\}$, and each $q \wedge s_{i} \in P \cap Q$, so that if $P \cap Q$ is closed, $q \wedge s \in P \cap Q \subseteq P$; and thus $s \in P$ since $P$ is prime and $q \notin P$.

Corollary 3. Let $P$ be a meet-irreducible set of prime subgroups of an l-group $H$ (i.e., $\cap \mathcal{P} \subset \cap \mathcal{P} \backslash\{P\}$ ) for every $P \in \mathcal{P}$ ). If $\cap \mathcal{P}$ is closed, so is every $P \in \mathscr{P}$.

We mention that the special case $\cap \mathscr{P}=\{1\}$ is a trivial consequence of a much stronger statement due to Lloyd [6, Theorem 2.3].

A convex subset $\Delta \neq \square$ of $\Omega$ is an o-block of $(G, \Omega)$ if, for every $g \in G$, either $\Delta g=\Delta$ or $\Delta g \cap \Delta=\square$. The convexification $\operatorname{Conv}(\bar{\Lambda})$ of $\bar{\Lambda} \subseteq \bar{\Omega}$ means $\left\{\omega \in \Omega \mid \bar{\lambda}_{1} \leq \omega \leq \bar{\lambda}_{2}\right.$ for some $\left.\bar{\lambda}_{1}, \bar{\lambda}_{2} \in \bar{\Lambda}\right\} \subseteq \Omega$. An o-block $\Delta$ of $G$ will be called extensive if Conv $\left(\bar{\delta} G_{\Delta}\right)=\Delta$ for one (hence every) $\bar{\delta} \epsilon \bar{\Delta}$. The special case of the following theorem in which $G$ is transitive (so that all o-blocks are exten- 
sive) and $\bar{\pi} \in \Omega$ was established by the author in [10, Theorem 11].

Theorem 4. Let $(G, \Omega)$ be an l-permutation group, and let $\bar{\pi} \in \bar{\Omega}$. An o-correspondence between the tower of nontrivial extensive o-blocks $\Delta$ of $(G, \Omega)$ for which $\bar{\pi} \in \bar{\Delta}$ and the tower of prime subgroups $C$ of $G$ for which $G_{\bar{\pi}} \subset C$ is given by $\Delta \rightarrow G_{\Delta}$ and $C \rightarrow \operatorname{Conv}(\bar{\pi} C)$.

Proof. [5, Theorem 3] states that nondisjoint o-blocks of tran sitive groups are comparable under inclusion, and the same proof works in the intransitive case for extensive o-blocks, so the lattice of o-blocks $\Delta$ for which $\bar{\pi} \in \bar{\Delta}$ is indeed totally ordered. If $\bar{\pi} \in \bar{\Delta}, \Delta$ an extensive ooblock, then $G_{\Delta}$ is clearly a prime subgroup of $G$ containing $G_{\bar{\pi}}$, and $\operatorname{Conv}(\bar{\pi} G)=\Delta$ since $\Delta$ is extensive. If $G_{\bar{\pi}}$ $C C, C$ prime, then $\Delta=\operatorname{Conv}(\bar{\pi} C)$ is an o-block of $G$ and $G_{\Delta}=C$. For if $\bar{\pi} c_{1} \leq \bar{\pi} c_{2} g \leq \bar{\pi} c_{3}$, then $c_{2} g \leq c_{2} g \vee c_{3} \in C$ since $\left(c_{2} g \vee c_{3}\right)_{c_{3}}{ }^{-1} \in G_{\bar{\pi}} \subseteq C$, and similarly $c_{2} g \geq c_{2} g \wedge c_{1} \in C$. Since $C$ is convex, $c_{2} g \in C$, so that $g \in C$ and $\Delta g=\Delta$, as required. Clearly $\Delta$ is extensive.

3. Closed subgroups of an l-permutation group. The author showed in [8, Theorem 7] that for a transitive l-subgroup $G$ of $A(\Omega)$, the following are equivalent:

(1) Sups in $G$ are pointwise, i.e., if in $G, g=\bigvee_{i \in I} g_{i}$, then, for each $\beta \epsilon$ $\Omega, \beta g$ is the sup in $\Omega$ of $\left\{\beta g_{i} \mid i \in I\right\}$.

(2) $G$ is a complete subgroup of $A(\Omega)$, i.e., if in $G, g=\bigvee_{i \in I} g_{i}$, then $g=$ $\bigvee_{i \in I} g_{i}$, also in $A(\Omega)$.

(3) $G_{a}$ is a closed subgroup of $G$ for all $a \in \Omega$. (Of course, if any $G_{\bar{\omega}}, \bar{\omega} \epsilon$ $\bar{\Omega}$, is closed, so is $G_{\bar{\sigma}}$ for every $\bar{\sigma} \in \bar{\omega} G_{\text {. }}$ )

Moreover, it was shown in [8, Corollary 15] that in the presence of these conditions, we have

(4) $G$ is a completely distributive l-group, i.e., $\bigwedge_{i \in I} \bigvee_{k \in K} g_{i k}=$ $\bigvee_{f \in K} \bigwedge_{i \in I} g_{i f(i)}$ for any collection $\left\{g_{i k} \mid i \in I, k \in K\right\}$ of elements of $G$ for which the indicated sups and infs exist.

Here we discuss these conditions without assuming transitivity.

Lemma 5. If $s=\sup \left\{s_{i}\right\}$ and $\sup \left\{\bar{\omega} s_{i}\right\}<\bar{\omega} s$ for some $\bar{\omega} \epsilon \bar{\Omega}$, this sup also fails to be pointwise for every $\bar{\tau}$ in the interval $\left(\left(\sup _{i}\left\{\bar{\omega} s_{i}\right\}\right)_{s^{-1}}, \bar{\omega}\right]$ of $\bar{\Omega}$.

Proof. $\sup \left\{\bar{\tau} s_{i}\right\} \leq \sup \left\{\bar{\omega} s_{i}\right\}<\bar{\tau} s$.

The support of $g \in A(\Omega)$ means $\{\omega \in \Omega \mid \omega g \neq \omega\}$, and any $\Delta \subseteq \Omega$ containing this set is said to support $g$. We let $\bar{\Omega}_{c}=\left\{\bar{\omega} \in \bar{\Omega} \mid G_{\bar{\omega}}\right.$ closed $\}$.

Lemma 6. If $\left\{s_{i}\right\} \subseteq G_{\bar{\omega}}, \bar{\omega} \in \bar{\Omega}$, and if $\sup \left\{s_{i}\right\}=s \notin G_{\bar{\omega}}$, then $G_{\bar{\tau}}$ is not closed for any $\bar{\tau} \in[\bar{\omega}, \bar{\omega} s)$, and this interval does not support any $1 \neq g \in G$. Hence $\bar{\Omega}_{c}$ is closed (in the order topology on $\bar{\Omega}$ ). 
Proof. We may assume without loss of generality that the $s_{i}{ }^{2} s$ are positive. Suppose by way of contradiction that $G_{\bar{\tau}}$ is closed for some $\bar{\tau} \epsilon[\bar{\omega}, \bar{\omega} s)$, and let $\bar{\sigma}=\bar{\tau} s^{-1}<\bar{\omega}$. Then $G_{\bar{\sigma}}$ is closed, and $\bar{\sigma} s<\bar{\sigma} s^{2}$, so that $\bar{\sigma}<\bar{\sigma} s$. Thus $\bar{\sigma}<$ $\overline{\sigma s}{ }_{j}<\bar{\omega}$ for some $s_{j}$, and letting $\bar{\rho}=\bar{\sigma} s_{j}, G_{\bar{\rho}}$ is closed. But $s_{i} s^{-1} s_{j} \vee 1 \in G_{\bar{\rho}}$ for each $i$ since $s_{i} \in G_{\bar{\omega}}$ guarantees that $\bar{\rho} s_{i} s^{-1} s_{j}<\bar{\omega} s^{-1} s_{j} \leq \bar{\tau} s^{-1} s_{j}=\bar{\sigma} s_{j}=\bar{\rho}$; whereas $\sup \left\{s_{i} s^{-1} s_{j} \vee 1\right\}=s_{j} \notin G_{\bar{\rho}}$, a contradiction. Hence $G_{\bar{T}}$ is not closed for any $\bar{\tau} \epsilon[\bar{\omega}, \bar{\omega} s)$. But then $G_{\bar{\tau}}$ is not closed for any $\bar{\tau} \in[\bar{\omega}, \bar{\omega} s) s^{-1}=$ $\left[\bar{\omega} s^{-1}, \bar{\omega}\right)$. Therefore, $\bar{\Omega} \backslash \bar{\Omega}_{c}$ is open in the order topology on $\bar{\Omega}$. Also, if $[\bar{\omega}, \bar{\omega} s)$ supports some $1 \neq g \in G$ (which can be assumed positive), then $s_{i} \leq$ $s g^{-1}<s$ for each $i$ ( since $\bar{\omega} s_{i}=\bar{\omega}$ ), a contradiction.

Theorem 7. For any l-permutation group, eacb condition (1)-(4) implies the next. Moreover,

(a) If sups in $G$ are pointwise (at points of $\Omega$ ), they are also pointwise at cuts of $\Omega$.

(b) If $G$ is complete in $A(\Omega)$ and transitive on $\Omega$, then $G$ is also complete in $A(\bar{\Omega})$.

(c) If stabilizers of points are closed, so are stabilizers of cuts.

Proof. The proof of the first statement is exactly as in [8]. (None of the implications can be reversed; a counterexample for the last one can be found in \$ 7.) (a) follows from Lemma 5 , and (c) from Lemma 6 . When $(G, \Omega)$ is transitive, (2) for $(G, \Omega)$ implies (1) for $(G, \Omega)$, which by (a) implies (1) for $(G, \bar{\Omega})$, which in turn implies (2) for $(G, \bar{\Omega})$, yielding (b); and (b) does in fact fail without transitivity.

A $G$-congruence $\mathfrak{I}$ is said to be convex if its congruence classes are convex. The corresponding partition of $\Omega$ into o-blocks of $G$ will al so be denoted by $\mathfrak{D}$. The set of o-blocks associated with a given $\mathscr{D}$ inherits a natural total order from $\Omega$, namely $\Delta_{1} \leq \Delta_{2}$ iff $\delta_{1} \leq \delta_{2}$ for some $\delta_{1} \in \Delta_{1}, \delta_{2} \in \Delta_{2}$. If $G$ is transitive, the o-blocks associated with $\mathfrak{D}$ are all translates of each other. In this case the partition $\mathscr{D}$ will be called an o-block system of $G$, and if $\Delta$ is an o-block, the system it determines will be denoted by $\widetilde{\alpha}$.

We shall say that $\bar{\omega} \epsilon \bar{\Omega}$ is a cut in $\mathscr{D}$ if $\bar{\omega}$ does not lie in the interior of $\bar{\Delta}$ for any $\Delta \in \mathscr{D}$. If $G$ is transitive and $\mathscr{D}$ nontrivial, then $\Delta \epsilon \mathfrak{D}$ lacks end points, so the same is true of $\bar{\Delta}$, and thus the condition reals " $\bar{\omega} \notin \bar{\Delta}$ for any $\Delta \epsilon \mathscr{D}$ ".

We shall say that a convex $G$-congruence $\mathfrak{D}$ is closed if for each $\mathscr{D}$-class $\Delta, G_{\Delta}\left(=G_{\text {sup } \Delta}=G_{\text {inf } \Delta}\right)$ is closed. If $\bar{\omega}$ is a cut in a closed $\mathscr{D}$, then $G_{\bar{\omega}}$ is closed (by definition if $\bar{\omega}$ is an end point in $\bar{\Omega}$ of some $\Delta \in \mathscr{D}$, and then in general by Lemma 6 ). If $G$ is transitive, and $\Delta$ is an o-block of $G$, then if $G_{\Delta}$ is

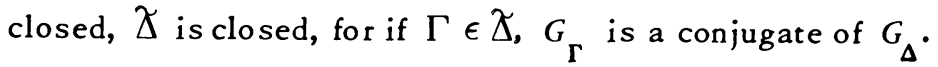


We partially ordered the collection of convex $G$-congruences by inclusion. In the transitive case, this gives a total order [5, Theorem 3].

Now we define an extremely important convex $G$-congruence $\mathcal{C}$ as follows: $\sigma \mathcal{C}_{\tau}$ iff either $\sigma=\tau$ or no $\bar{\delta} \epsilon \bar{\Omega}$ lying between $\sigma$ and $\tau$ (inclusive) has a closed stabilizer $G_{\bar{\delta}}$. Since $\bar{\Omega}_{c}=\left\{\bar{\omega} \in \bar{\Omega} \mid G_{\bar{\omega}}\right.$ is closed $\}$ satisfies $\bar{\Omega}_{c} g=\bar{\Omega}_{c}$ for every $g \in G, \mathcal{C}$ is indeed a (convex) $G$-congruence.

Theorem 8. Let $(G, \Omega)$ be an l-permutation group. Then

(1) $C$ is the smallest closed convex $G$-congruence, and a convex G-congruence $\mathfrak{D}$ is closed iff $\mathfrak{D} \geq \mathcal{C}$.

(2) $\mathcal{C}$ is the trivial system (of singletons) iff all stabilizers of points in $\Omega$ are closed.

(3) The C-classes are extensive o-blocks of $G$.

(4) $G_{\bar{\omega}}$ is closed iff $\bar{\omega}$ is a cut in $\mathcal{C}$, i.e., $\bar{\Omega}_{c}$ is the set of cuts in $\mathcal{C}$.

(5) If $\bar{\omega} \in \bar{\Delta}, \Delta \in \mathcal{C}$, then $G_{\bar{\omega}}^{*}=G_{\Delta}$.

(6) If $\bar{\omega}$ is a cut in $\mathcal{C}$ and $G_{\bar{\omega}}$ fixes $\bar{\tau}$, then $\bar{r}$ is also a cut in $\mathcal{C}$.

Proof. If $\Delta$ is a $C$-class, then every interval $[\sup \Delta, \omega)$ meets $\bar{\Omega}_{c}$, so by Lemma $6, G_{\text {sup } \Delta}$ is closed. Hence $\mathcal{C}$ is closed. (4) now follows immediately from the construction of $\mathcal{C}$. Hence if $\mathfrak{D} \geq \mathcal{C}, \mathfrak{D}$ is closed; and conversely, if $\mathfrak{D}$ is closed, then for any $\Delta \in \mathscr{D}, G_{\text {sup } \Delta}$ and $G_{\text {inf } \Delta}$ are closed, so that sup $\Delta$ and inf $\Delta$ are cuts in $\mathcal{C}$, and thus $\mathfrak{D} \geq \mathcal{C}$. This proves (1).

$\mathcal{C}$ is trivial iff $\bar{\Omega}_{c}$ is dense in $\bar{\Omega}$ iff $\bar{\Omega}_{c}=\bar{\Omega}$ (since $\bar{\Omega}_{c}$ is closed by Lemma 6) iff all point stabilizers are closed (by (a) of Theorem 7). If $\delta \in \Delta \in \mathcal{C}$, then Conv $\left(\delta G_{\Delta}\right)$ has the same (closed) stabilizer as $\Delta$, so its end points cannot lie in $\bar{\Delta}$, proving that $\Delta$ is extensive.

If $\bar{\omega}$ is not a cut in $\mathcal{C}$, so that $\bar{\omega} \in \bar{\Delta}, \Delta \in \mathcal{C}$, then by Theorem 4 , the tower of primes properly containing $G_{\bar{\omega}}$ is $\left\{G_{\Gamma} \mid \bar{\omega} \in \bar{\Gamma}, \Gamma\right.$ an extensive o-block of $\left.G\right\}$, and by (4), $G_{\Delta}$ is the smallest closed member of this tower, so that $G_{\bar{\omega}}^{*}=G_{\Delta}$. For (6), if $G_{\bar{\omega}}$ fixes $\bar{\tau}$, then $G_{\bar{\omega}} \subseteq G_{\bar{\tau}}$. Now if $\bar{\omega}$ is a cut in $\mathcal{C}$, so that $G_{\bar{\omega}}$ is closed, then $G_{\bar{\tau}}$ is also closed by Lemma 1 , so that $\bar{\tau}$ is also a cut in $\mathcal{C}$.

Our main theorem extends a line of thought begun in [9]. For transitive groups, it will be sharpened somewhat in Theorem 12. The closure of a convex l-subgroup $C$ will be denoted by $C^{*}$, and $\{\bar{\omega} \in \bar{\Omega} \mid \bar{\omega} C=\bar{\omega}\}$ by $\bar{F} x C$ (written without the bar in [9]). If $\bar{\Gamma} \subseteq \bar{\Omega}, G(\bar{\Gamma})$ will denote $\{g \in G \mid \overline{\gamma g}=\bar{\gamma}$ for each $\bar{\gamma} \in \bar{\Gamma}\}=$ $\bigcap\left\{G_{\bar{\gamma}} \mid \bar{\gamma} \in \bar{\Gamma}\right\}$. If $\mathfrak{D}$ is a convex $G$-congruence, we let $L(\mathscr{D})$ be the $l$-ideal $\{g \in G \mid \Delta g=\Delta$ for all $\Delta \in \mathscr{D}\}=\bigcap\left\{G_{\bar{\omega}} \mid \bar{\omega}\right.$ is a cut in $\left.\mathfrak{T}\right\}$. If $\mathfrak{T}$ is closed, $L(\mathfrak{D})$ is closed. If $\mathfrak{D}<\mathcal{E}$, then $L(\mathfrak{D}) \subseteq L(\mathcal{E})$. We let $G$ act on $\mathfrak{D}$ by defining $\Delta g \in \mathfrak{D}$ to be $\Delta g \subseteq \Omega$. This action is faithful iff $L(\mathscr{D})=\{1\}$. The distributive radical $D(G)$ of $G$ is the intersection of all the closed primes of $G$, and is $\{1\}$ iff $G$ is completely distributive [2, Theorem 3.4 and Corollary 3.8]. 
Main Theorem 9. Let $(G, \Omega)$ be an l-permutation group.

(1) If $C$ is any convex l-subgroup of $G$, then $C^{*} \supseteq G(\bar{F} \times C)$.

(2) If $C$ is any closed convex l-subgroup of $G, C=G(\bar{F} x C)=\bigcap\left\{G_{\bar{\delta}} \mid C \subseteq G_{\bar{\delta}}\right\}$; and if $C$ is the intersection of a finite collection of stabilizers, all stabilizers containing $C$ are closed.

(3) If the stabilizers of points of $\Omega$ are closed, the closed convex l-subgroups of $G$ are precisely the $G(\bar{\Delta})^{\prime} s, \bar{\Delta} \subseteq \bar{\Omega}$, and thus are intersections of closed primes.

(4) If $P$ is any prime subgroup of $G, \bar{F} x P^{*}=\bar{F} x P \cap \bar{\Omega}_{c}$ and $P^{*}=$ $G\left(\bar{F} \times P \cap \bar{\Omega}_{c}\right)$.

(5) If $P$ is any closed prime subgroup of $G$, the tower of primes $Q$ such that $P \subset Q \subset G$ consists entirely of closed stabilizers $G_{\bar{\omega}}$, and $P$ is a minimal closed prime unless $G_{\bar{\tau}} \subset P$ for some closed $G_{\bar{\tau}}$.

(6) In (5), if $P \neq G$ is not itself a stabilizer, then $P$ bas no cover in the tower of primes which contain it, and thus is an intersection of closed stabilizers; and $P$ is a minimal closed prime.

(7) The distributive radical $D(G)$ is $L(\mathcal{C})$.

(8) Every closed l-ideal of $G$ is $L(\mathfrak{D})$ for some $\mathfrak{D}$.

Proof. The proof of [9, Theorem 3] proves (1), which gives the first part of (2), which, in view of Theorem 7, gives (3). For the second part of (2), if $C$ is the intersection of a finite collection of stabilizers, it is the intersection of a (finite) meet-irreducible subcollection $\left\{G_{\bar{\gamma}} \mid \bar{\gamma} \in \bar{\Gamma}\right\}$, and the $G_{\bar{\gamma}}$ 's are closed by Corollary 3. Now let $C \subseteq G_{\bar{\delta}}$. If $G_{\bar{\delta}}$ is not closed, then for each $\bar{\gamma}, G_{\bar{\gamma}} \unrhd G_{\bar{\delta}}$ by Lemma 1, so there exists $1<g_{\bar{\gamma}} \epsilon G_{\bar{\gamma}}$ such that $\overline{\delta g}>\bar{\delta}$. Now $\wedge_{g_{\bar{\gamma}} \epsilon}$

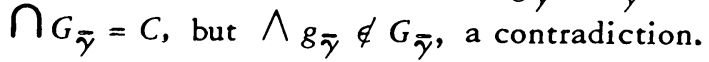

If $P$ is any prime subgroup of $G$, then $P^{*} \subseteq \bigcap\left\{G_{\bar{\omega}} \mid P \subseteq G_{\bar{\omega}}, \bar{\omega} \in \bar{\Omega}_{c}\right\}$ since the latter is closed, so that $\bar{F} x P^{*} \supseteq \bar{F} x P \cap \bar{\Omega}_{c}$. But of course $\bar{F} x P^{*} \subseteq \bar{F} x P$, and since $P^{*} \subseteq G_{\bar{\omega}}$ and $P^{*}$ closed implies $G_{\bar{\omega}}$ closed by Lemma 1 , we have $\bar{F} x P^{*} \subseteq$ $\bar{\Omega}_{c}$, so that $\bar{F} x P^{*}=\bar{F} x P \cap \bar{\Omega}_{c}$. By (1), $P^{*}=G\left(\bar{F} x P^{*}\right)=G\left(\bar{F} \times P \cap \bar{\Omega}_{c}\right)$.

Now let $P \neq G$ be a closed prime and let $\mathcal{T}$ be the tower of primes $Q$ (automatically closed by Lemma 1) such that $P \subset Q \subset G$. Let $C \in \mathcal{T}$. If $P$ is not a stabilizer, then since $P$ is the intersection of the stabilizers which contain it (by (2)), $\mathcal{T}$ has no smallest member (proving part of (6)) and the subset of $\mathcal{T}$ consisting of stabilizers is coinitial with $\mathcal{T}$. Thus in any case, $C$ contains a stabilizer $G_{\bar{\pi}}$. By Theorem $4, \Delta=\operatorname{Conv}(\bar{\pi} C)$ is an ooblock of $G$ and $G_{\Delta}=C$. Hence $C=G_{\text {sup }} \Delta^{*}$ This establishes the first part of (5), which, together with the part of (6) already proved, yields the rest of (5) and (6).

The distributive radical $D(G)$ is the intersection of the closed primes of $G$, and by (6), every closed prime is an intersection of closed stabilizers, so that $D(G)=\bigcap\left\{G_{\bar{a}} \mid \bar{\omega} \in \bar{\Omega}_{c}\right\}=L(\mathcal{C})$. 
Finally, let $C$ be any closed l-ideal of $G$. Since $C$ is normal in $G, \bar{F} \times C$ is a union of orbits of $(G, \bar{\Omega})$. Hence we obtain a convex $G$-congruence $\mathscr{D}$ by setting $\sigma D_{\tau}$ iff either $\sigma=\tau$ or no $\bar{\delta}$ between $\sigma$ and $\tau$ (inclusive) lies in $\bar{F} x C$. By (2), $C=G(\bar{F} \times C)=L(\mathscr{D})$. This concludes the proof.

Theorem 10. Let $(G, \Omega)$ be an l-permutation group. The following are equivalent:

(1) $G$ is completely distributive.

(2) G acts faithfully on $\mathcal{C}$.

(3) $C^{*}=G\left(\bar{F} \times C \cap \bar{\Omega}_{c}\right)$ for every convex l-subgroup $C$ of $G$.

(4) Every closed convex l-subgroup $C$ of $G$ is the intersection of the closed stabilizers which contain it.

Proof. $G$ is completely distributive iff $D(G)=\{1\}$, and $D(G)=L(C)$ by Theorem 9, so (1) and (2) are equivalent. In any completely distributive l-group, every closed convex l-subgroup is the intersection of the closed primes which contain it [9, Corollary 5], and hence, by (6) of Theorem 9, of the closed stabilizers which contain it. Thus (1) implies (4). Now suppose (4) holds, and let $C$ be a convex l-subgroup of $G$. Then if $G_{\bar{\omega}}$ is closed, $C^{*} \subseteq G_{\bar{\omega}}$ iff $C \subseteq G_{\bar{\omega}}$, so by (4), $C^{*}=$ $\bigcap\left\{G_{\bar{\omega}} \mid C^{*} \subseteq G_{\bar{\omega}}\right.$ and $G_{\bar{\omega}}$ closed $\}=\bigcap\left\{G_{\bar{\omega}} \mid \bar{\omega} \epsilon \bar{F} \times C \cap \overline{\bar{\Omega}}_{c}\right\}=G\left(\bar{F} \times C \cap \bar{\Omega}_{c}\right)$, proving (3). Finally, if (3) holds, then since $\bar{F} \times\{1\}=\bar{\Omega}$, we have $\{1\}=\{1\}^{*}=$ $G\left(\bar{\Omega}_{c}\right)=L(\mathcal{C})$, which is (2).

A representation of an l-group $H$ on a chain $\Sigma$ is an loisomorphism $\theta$ of $H$ into $A(\Sigma) . \theta$ is complete if it preserves arbitrary sups that exist in $H$, or equivalently, if $H \theta$ is a complete losubgroup of $A(\Sigma) . H$ has a complete representation iff $H$ is completely distributive [2, Theorem 3.10].

By the normal kernel of a convex losubgroup $C$ of an l-group $H$ we mean the intersection of all the conjugates of $H$, which is the largest $l$-ideal of $G$ contained in $C$. A collection $\left\{K_{\lambda} \mid \lambda \in \Lambda\right\}$ of primes of $H$ whose intersection has trivial normal kernel is called a Holland kernel of $H$. These kernels were used by Holland to obtain representations of $H$ as $l$-permutation groups [3, Theorem 2].

Corollary 11. Let $\left\{K_{\lambda} \mid \lambda \in \Lambda\right\}$ be a Holland kernel of an abstract l-group $H$, so that the normal kernel of $\cap K_{\lambda}$ is $\{1\}$. Then the normal kernel of $\bigcap_{K_{\lambda}^{*}}^{*}$ is $D(H)$. Hence if $H$ is completely distributive, $\left\{K_{\lambda}^{*}\right\}$ is also a Holland kernel.

Proof. Use the Holland representation to represent $H$ as an lopermutation group on a chain $\Omega$ so that each $K_{\lambda}$ is the stabilizer of some point $\omega_{\lambda}$ and the union of the orbits $\omega_{\lambda} H$ is $\Omega$. Then by Theorem 9 , the $K_{\lambda}^{*}$ 's are stabilizers of o-blocks in the smallest convex $H$-congruence $C$. Hence the collection of all conjugates of $K_{\lambda}^{*}$, $s$ is precisely the collection of stabilizers of all o-blocks in $\mathcal{C}$, and the intersection of these is $L(\mathcal{C})$, which by Theorem 9 is $D(H)$. 
4. Transitive $l$-permutation groups. Recall that in a transitive group $(G, \Omega)$, all the o-blocks of a given convex $G$-congruence are translates of each other, and that the o-block systems form a tower, so that the concept of the smallest closed system $\mathcal{C}$ is particularly nice. Further, for any $\omega \in \Omega$, the map $\Delta \rightarrow \mathcal{X}$ is an o-isomorphism from the tower of o-blocks $\Delta$ containing $\omega$ onto the tower of o-block systems.

Theorem 12. Let $(G, \Omega)$ be a transitive l-permutation group. Then

(1) $\mathcal{C}$ is the trivial system iff $G$ is a complete subgroup of $A(\Omega)$.

(2) The closed l-ideals of $G$ form a tower under inclusion.

(3) If $G$ bas only a finite number of closed o-block systems, every closed prime $\neq G$ is $G_{\bar{\omega}}$ for some cut $\bar{\omega}$ in $\mathcal{C}$ (i.e., (6) of Theorem 9 cannot occur).

(4) If $G$ is completely distributive, the closed l-ideals of $G$ are precisely the $L(D)$ 's.

Proof. $\mathcal{C}$ is the trivial system iff stabilizers of points are closed, and by [8, Theorem 7] this holds iff $G$ is complete in $A(\Omega)$. (2) follows from (8) of Theorem 9 since the o-block systems form a tower.

For (3), let $q$ be the number of closed o-block systems, and suppose that $P$ is as in (6) of Theorem 9, and thus has no prime cover. Then there exists above $P$ a stabilizer $G_{\bar{\omega}}$ above which there lies a tower of $q+1$ distinct closed stabilizers. Theorem 4 supplies a tower of $q+1$ distinct o-blocks whose systems are closed, a contradiction.

By Theorem 9, every closed $l$-ideal is $L(D)$ for some $\mathscr{D}$; and if $\mathfrak{D} \geq \mathcal{C}$, then $\mathfrak{D}$ and hence $L(\mathfrak{D})$ are closed. If $\mathfrak{D}<\mathcal{C}$, then $L(\mathscr{D})=L(\mathcal{C})=\{1\}$ if $G$ is completely distributive, completing the proof.

Question. Does the conclusion of (4) hold even without complete distributivity? Nontrivial $L(\mathscr{D})$ 's can be closed even when $\mathscr{D}$ is not, and perhaps they always are.

A representation $\theta$ of an $l$-group $H$ on a chain $\Sigma$ is transitive if $H \theta$ is trans itive on $\Sigma$. A representing subgroup of $H$ is a one element Holland kernel, i.e., a prime subgroup which contains no nontrivial $l$-ideal of $H$. If $H$ has a represento ing subgroup $K$, then the Holland representation obtained from $K$ is transitive and has $K$ as the stabilizer of a point; and conversely, if $(G, \Omega)$ is transitive, the stabilizers of points are representing subgroups of $G$. Thus if $H$ has a complete transitive representation, $H$ is completely distributive and has a representing subgroup. Conversely, we have

Corollary 13. Let $H$ be a completely distrubutive abstract l-group. Then

(1) If $\theta$ is a transitive representation of $H$ on a chain $\Sigma$, then $(H \theta, \Sigma)$ can be faithfully "reduced" to the complete transitive group $(H \theta, \mathcal{C})$, where $\mathcal{C}$ is the smallest closed system of $(H \theta, \Sigma)$. 
(2) If $K$ is a representing subgroup of $H$, so is $K^{*}$. Hence maximal representing subgroups are closed.

Proof. Since $H \theta$ is completely distributive, its action on $\mathcal{C}$ is faithful, and the stabilizers in this action are closed in $G$, so $G$ is complete in $A(\mathcal{C})$, proving (1). Since a representing subgroup is precisely a one element Holland kernel, (2) follows from Corollary 11.

A transitive group $(G, \Omega)$ is said to be weakly o-primitive [4] if $L(\mathscr{D})=\{1\}$ only when $\mathfrak{D}$ is the trivial system, so that $(G, \Omega)$ has no proper faithful reductions.

Corollary 14. Conditions (1), (2), (3), and (4) of Theorem 7 are equivalent for weakly o-primitive groups.

Proof. We need only show that (4) implies (3). But by Theorem 10, complete distributivity implies $L(\mathcal{C})=\{1\}$, so that by weak o-primitivity, $\mathcal{C}$ must be the trivial system, i.e., (3) holds.

Corollary 15. Let $H$ be an abstract l-group having a representing subgroup. Then the closed l-ideals of $H$ form a tower under inclusion.

Proof. Since $H$ has a transitive representation, we may use part (8) of Theorem 9.

6. The support property. If an o-block $\Delta$ of a transitive group $G$ supports

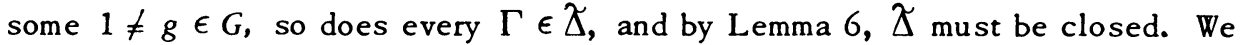
shall say that $G$ has the support property if each of its nontrivial o-blocks supports some $1 \neq g \in G$. Clearly groups having the support property are weakly oprimitive.

A transitive group $(G, \Omega)$ is called locally o-primitive if it has a smallest nontrivial o-block system $\mathfrak{D}$. In this case the o-blocks of $\mathcal{D}$ are called primitive segments. The $\left(G_{\Delta} \mid \Delta, \Delta\right)$ 's for the various primitive segments $\Delta$ are all isomorphic as l-permutation groups, and are ooprimitive; and a property enjoyed by them is said to be enjoyed locally by $(G, \Omega)$. If $G$ is not locally ooprimitive, then $\{a\}=\bigcap\{\Delta \mid a \in \Delta, \Delta$ a nontrivial o-block $\}$, so that the support property implies that every nondegenerate interval of $\Omega$ supports some $1 \neq g \in G$. In view of the next lemma, this implication also holds if $G$ is locally nonpathologically $o-2-t r a n s-$ itive. (An 0 -2-transitive group is pathological if it lacks elements $\neq 1$ of bounded support.)

Lemma 16. Let $(G, \Omega)$ be a locally o-primitive group having the support property. Let $\Delta$ be a primitive segment, let $K=G_{\Delta} \mid \Delta$, and let $L=\{g \mid \Delta: \Delta$ supports g\}. Then if $G$ is locally o-2-transitive, $L$ is an o-2-transitive l-ideal of $K$ containing all elements of bounded support; and otherwise $L=K$. 
Proof. It is easily checked that $L$ is an loideal of $K$, and the support property states that $L \neq\{1\}$. In pathologically 0 -2-transitive groups, all nontrivial l-ideals are (pathologically) o-2-transitive [11, Theorem 6], and in nonpathologically $0-2-t r a n s i t i v e$ groups, the $0-2-t$ ransitive loideal consisting of the elements of bound ed support is contained in every nontrivial loideal (by the proof of [3, Theorem 6]).

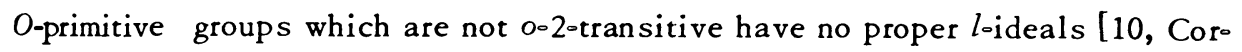
ollary 46]. The lemma follows.

The following theorem generalizes [12, Theorem 4], which states that an 0 primitive group fails to be complete iff it is pathologically o-2-transitive.

Theorem 17. Let $(G, \Omega)$ be a transitive l-permutation group baving the support property. Then $(G, \Omega)$ fails to be complete if and only if it is locally pathologically o-2-transitive; and in this case, the smallest closed o-block system $\mathcal{C}$ is the smallest nontrivial system.

Proof. Since by Lemma 6 the support by an o-block $\Delta$ of some $1 \neq g \in G$

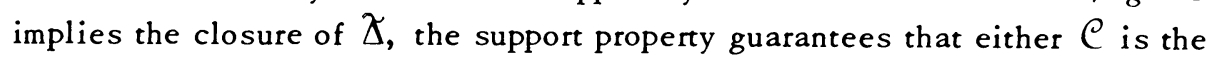
trivial system (i.e., $G$ is complete), or $G$ is locally ooprimitive and $C$ is the smallest nontrivial system. It remains to show that when $G$ is locally ooprimitive, the stabilizers $G_{a}$ fail to be closed iff $G$ is locally pathologically $0-2$-tran sitive.

Let $a \in \Omega$, let $\Delta$ be the primitive segment containing $\alpha$, and let $K$ and $L$ be as in the lemma. Suppose $s=\sup \left\{s_{i}\right\}$, with each $s_{i} \in G_{a}$ and $s \in G \backslash G_{a^{*}}$. Since $G_{\Delta}$ is closed, as $\epsilon \Delta,(K, \Delta)$ is ooprimitive, and if it is not pathologically $0-2$-transitive, $K_{a}$ is closed in $K[12$, Theorem 4]. Thus, letting $\hat{s}=s \mid \Delta$ and $\hat{s}_{i}=s_{i} \mid \Delta$, there exists $1<\hat{t} \in K$ such that, for each $i, \hat{s}_{i} \leq \hat{s} \hat{t}^{-1}<\hat{s}$. By the lemma, $\hat{t} \in L$. (If $(K, \Delta)$ is nonpathologically $0 \cdot 2$-transitive, $\hat{t}$ exceeds some $1<$ $k \in K$ having bounded support, so we may assume that $\hat{t}$ has bounded support.) Hence $\Delta$ supports some $t \in G$ such that $t \mid \Delta=\hat{t}$. $s t^{-1}$ agrees with $\hat{s} \hat{t}^{-1}$ on $\Delta$ and with $s$ outside $\Delta$, so for each $i, s{ }_{i} \leq s t^{-1}<s$, a contradiction. Therefore $G_{a}$ is closed in $G$.

Conversely, suppose that $G$ is locally pathologically o-2-transitive. By the lemma, $L$ is (pathologically) o-2otransitive, so there exists $\left\{\hat{s}_{i}\right\} \subseteq L_{a}$ having in $L$ a sup $\hat{s} \notin L_{a^{*}} \Delta$ supports $s, s_{i} \in G$ such that $s \mid \Delta=\hat{s}$ and $s_{i} \mid \Delta=\hat{s}_{i^{*}}$ Then $s=\sup \left\{s_{i}\right\}$ in $G$, for if $s_{i} \leq t<s$, then $\Delta$ supports $t$, so that $t \mid \Delta$ yields a contradiction. Therefore $G_{a}$ is not closed in $G$.

If an l-permutation group $(G, \Omega)$ has the support property and is locally o-primitive, but not locally pathologically $0^{\circ} 2-\operatorname{tran}$ sitive, the other transitive representations of $G$ can be very precisely described. (The description is due to Holland [4, Theorem 7]. In [11, Theorem 7], the hypotheses are relaxed to those just meno tioned, and it is shown that pathologically o-2otransitive groups need not satisfy 
the description.) Theorem 17 says that the groups $(G, \Omega)$ satisfying these hypotheses are precisely those locally o-primitive groups having the support property which are complete.

Corollary 18. Let $\left\{\left(G_{\gamma}, \Omega_{\gamma}\right) \mid \gamma \in \Gamma\right\}$ be a collection of transitive l-permutation groups indexed by a chain $\Gamma$, and let $(W, \Omega)$ be its ordered wreath product $[5, \S 3]$. The transitive l-permutation group $(W, \Omega)$ fails to be complete if and only if $\Gamma$ has a least element 0 and $\left(G_{0}, \Omega_{0}\right)$ is pathologically o-2-transitive.

7. Fxamples. Our first example shows that completely distributive transitive groups need not have closed stabilizers (cf. Theorem 7 and Corollary 14). Holland $[4$, p. 433] pointed out that if $\Omega$ is the chain of real numbers, the stabilizer subgroups of $A(\Omega)$ are not minimal representing subgroups. Pick a representing subgroup $K$ properly contained in a stabilizer. By part (3) of Theorem 12, $K$ is not closed. Thus in the Holland representation of $A(\Omega)$ on $R(K), K$ is the stabilizer of a point and is not closed, even though $A(\Omega)$ is completely distributive.

Next, we exhibit a transitive group $(G, \Omega)$ with $G$ totally ordered, so that $\{1\}$ is a closed prime, but with no stabilizer $G_{\bar{\omega}}=\{1\}$ (cf. Theorem 9). Let $I$ be the o-group of integers. Let $J$ be the o-group

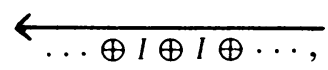

the small direct sum (indexed by $I$ ) of copies of $I$, ordered lexicographically from the right. Let $f$ be the o-group automorphism of $J$ which shifts each sequence one place to the right. Map $1 \in I$ onto $f$ and extend the map to a group homomorphism of $I$ into the group of o.group automorphisms of $J$. Take $G$ to be the semidirect sum $\overleftarrow{J \oplus I}$ (i.e., $\left.\left\langle j_{1}, i_{1}\right\rangle+\left\langle j_{2}, i_{2}\right\rangle=\left\langle j_{1} f^{i}+j_{2}, i_{1}+i_{2}\right\rangle\right)$, ordered lexicographically from the right to give an o-group. Let $K=\{\langle j, 0\rangle \mid j(n)=0$ for all $n \geq 0\}$. $K$ is a representing subgroup of $G$, for if $\langle 0,0\rangle \neq\langle j, 0\rangle \in K$, and if $n<$ 0 is the greatest integer such that $j(n) \neq 0$, then $\langle 0, n\rangle+\langle j, 0\rangle+\langle 0,-n\rangle \notin K$. Let $G$ act on $\Omega=R(K)$ via the Holland representation. Letting $\alpha=K$, we have $K+\langle j, i\rangle \in F x G_{a}$ iff $i \geq 0$, so that $F x G_{a}$ is a cofinal segment of $\Omega$ extending properly below $\alpha$. For any $\bar{\omega} \in \bar{\Omega}$, we can pick $\beta<\bar{\omega}$, and then $\{1\} \neq G_{\beta} \subseteq G_{\bar{\omega}}$. Therefore $\{1\}$ is not a stabilizer $G_{\bar{\omega}}$.

The argument that $K$ is a representing subgroup also shows that $K$ is prop-. erly contained in certain of its conjugates. (In [10, $\$ 3]$, groups exhibiting this pathology were called imbalanced, but the present example, which had been pointed out to the author by Charles Holland, was omitted for the sake of brevity.) Therefore, $K\left(=G_{a}\right)$ is not a maximal representing subgroup, so that $(G, \Omega)$ is not weakly ooprimitive. However, $G_{a}$ is closed in $G$ since $G$ is an oogroup, so that $(G, \Omega)$ is complete. Thus among completely distributive transitive groups, weak o-primitivity is properly stronger than completeness (cf. Corollary 14). 
Closed primes which are not stabilizers can occur even in groups which are complete and weakly and locally o-primitive: Form an ordered wreath product $(W, \Sigma)$ of two factors, with the previous group $(G, \Omega)$ as top factor, and $(A(\Lambda), \Lambda)$, $\Lambda$ the reals, as bottom factor. $W$ is complete by Corollary 18 . But $L(\mathscr{D}), \mathfrak{D}$ the smallest nontrivial o-block system, is a closed prime (since $G$ is an orgroup) which is not a stabilizer.

\section{REFERENCES}

1. R. D. Byrd, Complete distributivity in lattice-ordered groups, Pacific J. Math. 20 (1967), 423-432. MR $34 \# 7680$.

2. R. D. Byrd and J. T. Lloyd, Closed subgroups and complete distributivity in latticeordered groups, Math. Z. 101 (1967), 123-130. MR 36 \#1371.

3. C. Holland, The lattice-ordered group of automorphisms of an ordered set, Michigan Math. J. 10 (1963), 399-408. MR 28 \#1237.

4. - Transitive lattice-ordered permutation groups, Math. Z. 87 (1965), 420-433. MR $31 \# 2310$.

5. W. C. Holland and S. H. McCleary, Wreath products of ordered permutation groups, Pacific J. Math. 31 (1969), 703-716. MR 41 \#3350.

6. J. T. Lloyd, Representations of lattice-ordered groups having a basis, Pacific J. Math. 15 (1965), 1313-1317. MR 32 \#2489.

7. - Complete distributivity in certain infinite permutation groups, Michigan Math. J. 14 (1967), 393-400. MR 36 \#2544.

8. S. H. McCleary, Pointwise suprema of order-preserving permutations, Illinois J. Math. 16 (1972), 69-75.

9. - The closed prime subgroups of certain ordered permutation groups, Pacific J. Math. 31 (1969), 745-753. MR 42 \#1736.

10. - O-primitive ordered permutation groups, Pacific J. Math. 40 (1972), 349-372.

11. - O-2-transitive ordered permutation groups (to appear).

12. - O-primitive ordered permutation groups. II (to appear).

DEPARTMENT OF MATHEMATICS, UNIVERSITY OF GEORGIA, ATHENS, GEORGIA 30601 